\title{
Microsatellite instability in Chinese gastric cancer and its correlation with clinical characteristics
}

\author{
Xinyu Li ${ }^{1 \#}$, Lan Zhang ${ }^{2 \#}$, Chunxiao Wang ${ }^{3 \#}$, Junli Huang ${ }^{4 \#}$, Jinfeng Zhu ${ }^{5}$, Yisen Huang ${ }^{6}$, Yifeng Chen ${ }^{7}$, \\ Jing Jia ${ }^{1} \wedge$, Guozhong Jiang ${ }^{2}$ \\ ${ }^{1}$ Department of Gastrointestinal Surgery, The First Hospital of Quanzhou Affiliated to Fujian Medical University, Quanzhou, China; ${ }^{2}$ Department of \\ Pathology, the First Affiliated Hospital of Zhengzhou University, Zhengzhou, China; ${ }^{3}$ Shanghai Tongshu Biotechnology Co., Ltd, Shanghai, China; \\ ${ }^{4}$ Department of General Surgery, Army 73rd Group Military Hospital of the Chinese People's Liberation Army (Chenggong Hospital of Xiamen \\ University), Xiamen, China; ${ }^{5}$ Department of Medical Oncology, The First Hospital of Quanzhou Affiliated to Fujian Medical University, Quanzhou, \\ China; ${ }^{6}$ Department of Gastroenterology, The First Hospital of Quanzhou Affiliated to Fujian Medical University, Quanzhou, China; ${ }^{7}$ Department \\ of Pathology, The First Hospital of Quanzhou Affiliated to Fujian Medical University, Quanzhou, China \\ Contributions: (I) Conception and design: All authors; (II) Administrative support: All authors; (III) Provision of study materials or patients: All \\ authors; (IV) Collection and assembly of data: All authors; (V) Data analysis and interpretation: All authors; (VI) Manuscript writing: All authors; (VII) \\ Final approval of manuscript: All authors. \\ \#These authors contributed equally to this work. \\ Correspondence to: Guozhong Jiang. Department of Pathology, The First Affiliated Hospital of Zhengzhou University, Zhengzhou 450000, China. \\ Email: guozhongjiang@zzu.edu.cn; Jing Jia. Department of Gastrointestinal Surgery, The First Hospital of Quanzhou Affiliated to Fujian Medical \\ University, Quanzhou 362002, China. Email: jingjia_01@163.com.
}

Background: Microsatellite instability (MSI) remains a focus of interest in cancer research, but the characteristics of MSI in gastric cancer (GC) are ambiguous.

Methods: In this retrospective study, we analyzed the prevalence of MSI and the expression of programmed death-ligand 1 (PD-L1) and cluster of differentiation 8 (CD8) cells in Chinese GC patients. A total of 393 GC patients admitted to two centers from January 2010 to December 2017 were enrolled.

Results: The prevalence of MSI in this cohort was 3.4\% and most frequently occurred in females, patients aged between 59 and 69 years, and patients at a lower clinical stage. All MSI GCs had CD8 expression but lacked PD-L1 expression, indicating that MSI was related to CD8 expression but irrelevant to PD-L1 expression. However, there was no significant difference in the expression of CD8/PD-L1 between MSI GC and microsatellite stable (MSS) GC. Kaplan-Meier survival curves revealed that patients with MSI had a significantly longer overall survival (OS) than patients with MSS.

Conclusions: In Chinese GC patients, MSI frequently occurred in females, patients aged between 59 and 69, and patients with lower clinical stages. Patients with MSI-High (MSI-H) and MSI-Low (MSI-L) had a longer OS than patients with MSS. MSI was related to CD8 expression but irrelevant to PD-L1 expression.

Keywords: Gastric cancer (GC); microsatellite instability (MSI); immunotype; prognosis; CD8 expression

Submitted Sep 16, 2021. Accepted for publication Dec 08, 2021.

doi: 10.21037/jgo-21-695

View this article at: https://dx.doi.org/10.21037/jgo-21-695

^ ORCID: 0000-0002-8525-7950. 


\section{Introduction}

Gastric cancer (GC) is one of the most common causes of cancer-related deaths globally (1). Surgical resection with adjuvant chemotherapy is widely acknowledged as an effective treatment for patients with early-stage GC (2-5). However, recurrence occurs in up to $30-40 \%$ of patients within 5 years, suggesting that GC is a clinically heterogeneous disease (6-8). According to Lauren's classification, GCs are classified into two histological types: intestinal and diffuse. Additionally, GCs are divided into papillary, tubular, mucinous (colloid), and poorly cohesive carcinomas by the World Health Organization (WHO) Classification $(9,10)$. However, these classification systems have poor clinical utility. The Cancer Genome Atlas (TCGA) Research Network team conducted a landmark study that reported comprehensive findings of genetic mutations associated with GC, combining data from different platforms (11). Based on the integrated analysis of molecular information, GC was divided into four new molecular subtypes with clear genetic characteristics, including chromosome instability (CIN) type, microsatellite instability (MSI) type, gene stable (GS) type, and EpsteinBarr virus (EBV) positive type (11). This study hypothesized that tumor classification based on molecular data has more clinical influence in predicting treatment and patient prognosis than traditional pathological classification (11). The prognostic significance of these four molecular GC subtypes was validated by Sohn et al., who found that patients with the MSI subtype were characterized by diagnosis at a median age of 60 years, a moderate prognosis, and a moderate benefit from adjuvant chemotherapy (12).

MSI refers to the phenomenon that the length of microsatellite sequence changes due to insertion or deletion mutations during DNA replication, often caused by mismatch repair (MMR) functional defects (13). Due to the large number of microsatellites and spread across the entire genome, the instability of microsatellites located on multiple genes will lead to the abnormal function of multiple genes in multiple signaling pathways related to tumors, which will lead to the occurrence and development of MSI-H tumors. The MSI phenomenon was first discovered in colorectal cancer by Jacobs et al. in 1993 (14). Several clinical trials, including the CLASSIC, MAGIC and ARTIST trials, indicated that GC with a high MSI (MSI-H) was correlated with a favorable prognosis (15-17). The meta-analysis of the prognostic role of MSI in patients with resectable GC enrolled in the MAGIC, CLASSIC, ARTIST, and ITACA-S trials showed that MSI was a robust prognostic marker that should be adopted as a stratification factor by clinical trials and investigated prospectively (18). Adjuvant chemotherapy benefited GCs with MSI-Low (MSI-L) and microsatellite stable (MSS) tumors but not those with MSI-H tumors $(15,16,18)$. MSI plays a role as a predictive biomarker for immunotherapy, and the Food and Drug Administration (FDA) has approved pembrolizumab for advanced GC with programmed death-ligand 1 (PD-L1)+ tumors or MSI-H tumors based on the results of KEYNOTE 059 (19-23). The PD-L1 expression percentage of TMB high and/or MSI-H samples will be higher than TMB low and/or MSS samples $(24,25)$. The work of Thompson et al. suggested that GCs with a higher cluster of differentiation $8(\mathrm{CD} 8)^{+}$ T-cell densities also have higher PD-L1 expressions, and this subset of patients may be the ones who will derive the most benefit from checkpoint inhibition (26). Some studies used TCGA gastric cancer data to analyze the correlation between MSI status and PD-L1 or CD8, but few Chinese people were included in the TCGA database (27).

Here, we aimed to study the prevalence of MSI status and CD8/PD-L1 expression in Chinese patients with GC and to evaluate the relationship between MSI status and CD8/PD-L1 expression, to provide further information for PD-L1 immunotherapy in clinical settings. We present the following article in accordance with the REMARK reporting checklist (available at https://dx.doi.org/10.21037/ jgo-21-695).

\section{Methods}

\section{Patients}

This multi-center retrospective study included 393 patients with GC admitted to The First Affiliated Hospital of Zhengzhou University (Henan Province, China) and The First Hospital of Quanzhou (Fujian Province, China) from 2010 to 2017. The participants were screened with the following criteria: GC confirmed by pathology; acceptance of radical or local surgical treatment; availability of detailed clinical and pathological data for statistical analysis, including age, sex, TNM stage, degree of differentiation, clinical stage, Lauren subtype, and the degree of infiltration. We collected paraffin-embedded tissues from each patient, including the tumor and adjacent normal tissues, and cut them into 5-micro-thick sections. This study was approved by the Ethics Committee of The First Hospital of Quanzhou (No. 2020-126). All participants provided written informed 
Table 1 Detection loci of NCI MSI panel loci ( $\mathrm{n}=5)$

\begin{tabular}{ll}
\hline Mononucleotide $(\mathrm{n}=2)$ & Dinucleotide $(\mathrm{n}=3)$ \\
\hline BAT-25 & D2S123 \\
BAT-26 & D5S346 \\
& D17S250 \\
\hline
\end{tabular}

$\mathrm{NCl}$, The National Cancer Institute; MSI, microsatellite instability.

consent. The study was conducted in accordance with the Declaration of Helsinki (as revised in 2013).

\section{PD-L1 and CD8 detection}

The expression of PD-L1 (22C3; Dako Inc., Carpinteria, California, USA) and CD8 (SP16; Gene Tech Co. Ltd., Shanghai, China) were detected by immunohistochemistry (IHC), assessed by two pathologists who were blinded to the clinical characteristics and outcomes of patients. The PD-L1 expression on tumor and immune cells was evaluated according to the National Comprehensive Cancer Network (NCCN) guideline: positive expression was defined as $\geq 1 \%$ of capsular polysaccharide (CPS) in tumor cells (TCs) or immune cells (ICs), and negative expression was defined as $<1 \%$ of CPS in TC or IC. The CD 8 expression on lymphocytes was reported as the proportion of positive cells in all nucleated cells in each nuclear compartment and was defined as negative $(\leq 10 \%)$ or positive $(\geq 10 \%)$.

\section{MSI detection}

DNA was extracted from the formalin-fixed, paraffinembedded sections using a DNA extraction kit (FD-50, Changzhou Tongshu Biotechnology Co. Ltd., China) according to the manufacturer's protocols and was eluted with $50 \mu \mathrm{L}$ of Tris buffer (pH 7.5). Subsequently, DNA was quantified on a Nanodrop 2000 (Thermo Fisher Scientific Inc, Massachusetts, USA). Samples with concentrations $>5 \mathrm{ng} / \mu \mathrm{L}$ were used.

An MSI test was performed on 377 specimens collected by capillary electrophoresis with the NCI MSI panel kit (Tongshu BioTech., Shanghai, China) (Table 1). This panel contains two mononucleotide loci (BAT25, BAT26), three dinucleotide loci (D2S123, D5S346, D17S250), and one pentanucleotide repeat marker (Penta $\mathrm{C}$ ) as the internal control.

Polymerase chain reaction (PCR) amplification was performed on an A200 Gradient Thermal Cycler
(LongGene, China) in a volume of $10 \mu \mathrm{L}$ containing $5 \mu \mathrm{L}$ $2 \times$ PCR Master Mix, $2 \mu \mathrm{L} 5 \times$ primer mix, $0.2 \mu \mathrm{L}$ Amplitaq Gold DNA polymerase ( 5 units $/ \mu \mathrm{L}$ ), and $5 \mathrm{ng}$ DNA templates. The Thermal Cycler conditions were as follows: $95^{\circ} \mathrm{C}$ for $4 \mathrm{~min}, 30$ cycles of $95^{\circ} \mathrm{C}$ for $30 \mathrm{sec}, 60^{\circ} \mathrm{C}$ for $30 \mathrm{sec}$, $72{ }^{\circ} \mathrm{C}$ for $30 \mathrm{sec}$, and $60{ }^{\circ} \mathrm{C}$ for $45 \mathrm{~min}$. PCR products were detected and analyzed by an ABI 3730 genetic analyzer (Applied Biosystems, CA, USA) following the manufacturer's protocol. Data analysis was performed using the GeneScan Analysis and Genotyper Software packages (Applied Biosystems, CA, USA). Finally, MSI status was determined by the number of allelic bases and the internal control index.

\section{Survival analysis}

Disease-free survival (DFS) was defined as the time from the day of surgery to local recurrence, distant metastasis, or the last follow-up. Patients who died or were lost to followup were processed with censored data. Overall survival (OS) was defined as the time from the day of surgery to death for any reason or the last follow-up. The overall follow-up time was 60 months.

\section{Statistical analysis}

Statistical analysis was performed using SPSS 21.0 (SPSS Inc., Chicago, IL, USA). A Chi-square test was applied to analyze the relationship between clinicopathologic data and MSI or MSS in GC. Survival analysis was estimated using the Kaplan-Meier method and Log-Rank test. A P value $<0.05$ was regarded as statistically significant.

\section{Results}

\section{Clinicopathologic characteristics of GC patients}

A total of 393 gastric cancer patients were enrolled in this study, and the patient samples were collected from Quanzhou (south China) and Zhengzhou (north China) hospitals. The number is really not enough to represent the MSI characteristics of the whole Chinese population. However, the characteristics of MSI in gastric cancer in China can be preliminarily studied and we will continue to collect gastric cancer samples for further research. According to the screening process (Figure 1), 16 patients were excluded after the tumor content assessment due to inadequate tumor cells for analysis. Finally, 377 patients were included for further analysis. The demographic and clinicopathological 


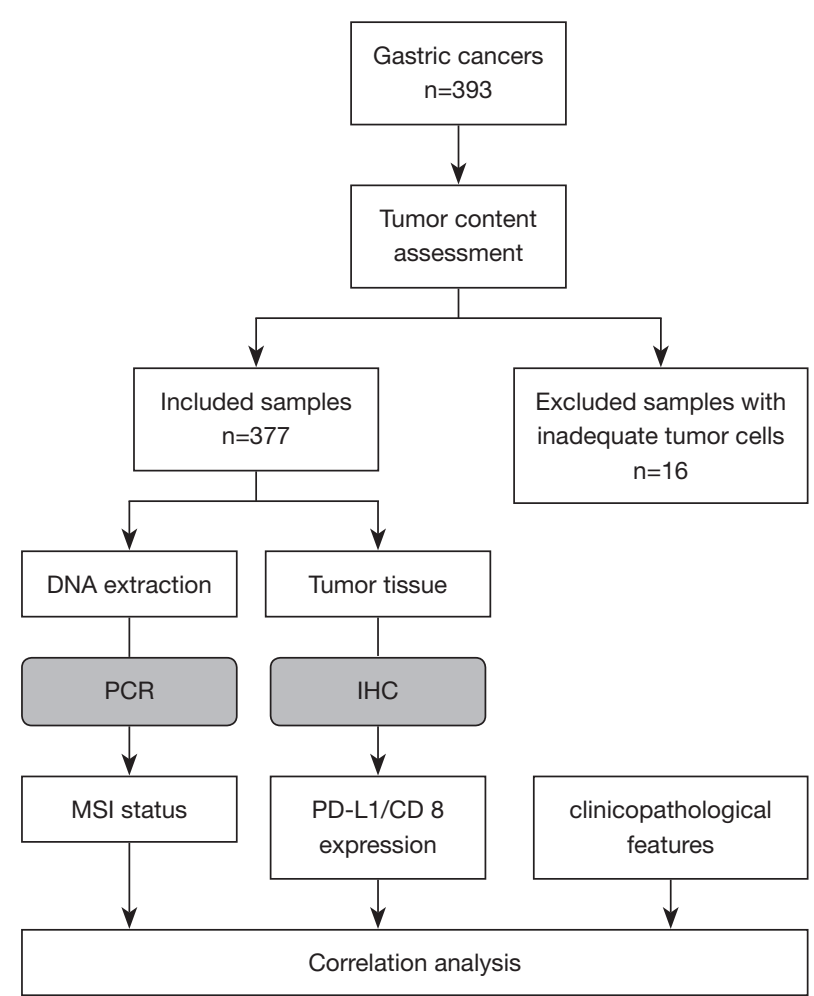

Figure 1 The flowchart of the screening process and the molecular testing. IHC, immunohistochemistry; PCR, polymerase chain reaction; MSI, microsatellite instability.

information of the enrolled patients is shown in Table 2.

Among the patients, there were 104 females and 273 males. The average age was $59 \pm 11.3$ years, the median age was 60 years, and 147 patients $(39.0 \%)$ were between 59 and 69 years. According to the TNM staging system, 51 patients were at lower stages (I or II), and 326 patients were at higher stages (III or IV); 115 patients (30.5\%) had lymph node metastasis, and 15 patients $(4.0 \%)$ had distant metastasis. The number of patients with low, medium, and high differentiation was 168,195 , and 14 , respectively. There were 107 patients at clinical stage I or II and 270 patients at clinical stage III or IV. In addition, there were 114 patients with the intestinal subtype, 155 with the diffuse subtype, and 108 patients were mixed.

\section{The correlation between MSI status and clinicopathological parameters in patients with GC}

In this cohort, $3.4 \%$ of patients were MSI-H/L, and $2.1 \%$ were MSI-H. The occurrence of MSI in females $(6.7 \%$, $7 / 104)$ was significantly higher than that in males $(2.2 \%$,
6/273) $(\mathrm{P}=0.031)$. The occurrence of MSI in patients aged from 59 to $69(6.1 \%, 9 / 147)$ was markedly higher than in other age groups $(1.7 \%, 4 / 230)(\mathrm{P}=0.023)$. The occurrence of MSI in patients with smaller tumors (T stage I-II, $7.8 \%$, $4 / 51$ ) was higher than that in patients with larger tumors (T stage III-IV, 2.8\%, 9/326), but no significant difference was observed $(\mathrm{P}=0.064)$. And the occurrence of MSI in lower clinical stages (I-II, 6.5\%, 7/107) was significantly higher than that in higher clinical stages (III-IV, 2.2\%, 6/270) $(\mathrm{P}=0.038)$. However, no difference was observed between patients with lymph node metastasis $(2.6 \%, 3 / 115)$ and GC patients without lymph node metastasis $(3.8 \%$, $10 / 262)$. The occurrence of MSI in patients with distant metastasis $(6.7 \%, 1 / 15)$ was higher than those without metastasis $(3.3 \%, 12 / 362)$, but there was no significant difference. MSI was not found in patients with high degrees of differentiation, and there was no significant difference between the various degrees of differentiation. Additionally, there was no difference in MSI occurrence among the different Lauren subtypes. Kaplan-Meier survival curves were generated according to the MSI status. The median overall survival (OS) of patients with MSI-H/MSI-L and MSS were 17.60 and 12.86 months, respectively. As shown in Figure 2, patients with MSI-H/MSI-L had a significantly longer OS compared with patients with MSS ( $\mathrm{P}=0.029)$.

\section{Clinical relevance of PD-L1 expression and $C D 8^{+} T$ cell infiltration in GC patients with MSI}

To evaluate the relationship between PD-L1 expression and the presence of $\mathrm{CD}^{+}$tumor-infiltrating lymphocyte (TIL), IHC was conducted to test PD-L1 expression and CD8 ${ }^{+}$TILs (Figure S1). The results of PD-L1 and CD8 expression are summarized in Table 3. In this cohort, only 12 patients exhibited PD-L1 expression, but none was MSI. However, 359 of 377 patients (95.2\%) had CD8 expression; moreover, all patients with MSI were CD8-positive. The relationship between PD-L1 and CD8 expression is shown in Table 4. We found that all patients with PD-L1 expression $(n=12)$ had CD8 expression, suggesting that CD8 plays a role in GC while PD-L1 does not.

\section{Discussion}

Microsatellites are the simple sequence repeats (SSR) of nucleotides in the genome and can be used for genetic markers, genetic linkage maps, and gene positioning. MSIs are SSR variations caused by replication errors, leading 
Table 2 The demographic and clinicopathological information of enrolled patients with gastric cancer

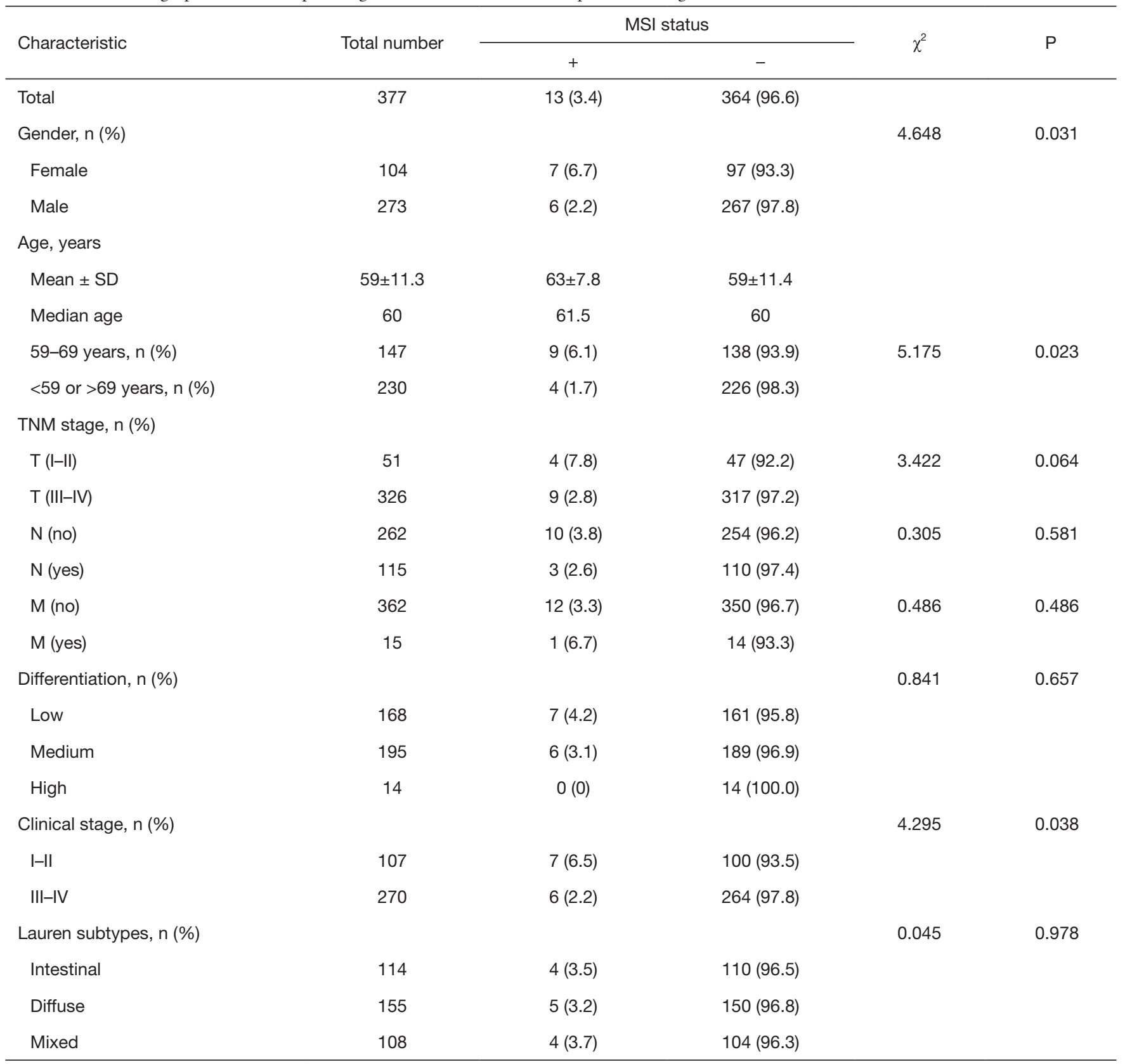

MSI, microsatellite instability; SD, standard deviation; T, tumor; $N$, lymph node; $M$, metastasis.

to tumorigenesis and tumor metastasis $(28,29)$. Previous studies on Western populations have reported that the rate of GC patients with MSI was $9.0-22.0 \%$, indicating that MSI could be used as a diagnostic marker of GC $(11,30,31)$. The prevalence of MSI in Chinese GC has been reported to be $2.3-8.0 \%$, which is consistent with our findings $(2.1 \%)$ and suggests that the prevalence of MSI in Chinese
GC patients is lower than that in Western GC patients (32-34). Furthermore, consistent with other studies, our results demonstrated that MSI was correlated with patients' clinicopathological features, including gender, age, tumor size, and clinical stage. Female patients between 59 and 69 years with a smaller tumor size or an earlier clinical stage were more likely to have an MSI status and have a more 
favorable prognosis than GC patients with an MSS status $(35,36)$. Due to the small sample size of GC patients with MSI in this study, the data distribution between MSI-H and MSS samples was biased (13 vs. 364), which may have led to biased statistical results. Therefore, we will need to include more MSI-H GC patients in future studies to confirm our present findings.

Previous studies have shown increased lymphocyte infiltration in the MSI tumor microenvironment (37). The results of clinical trials of anti-PD-1/PD-L1 drugs suggested that patients with MSI tumors could obtain a better clinical response and a prolonged survival time compared with patients with MSS tumors, which may be related to the expression of various immune molecules in the MSI tumor microenvironment $(38,39)$. In addition,

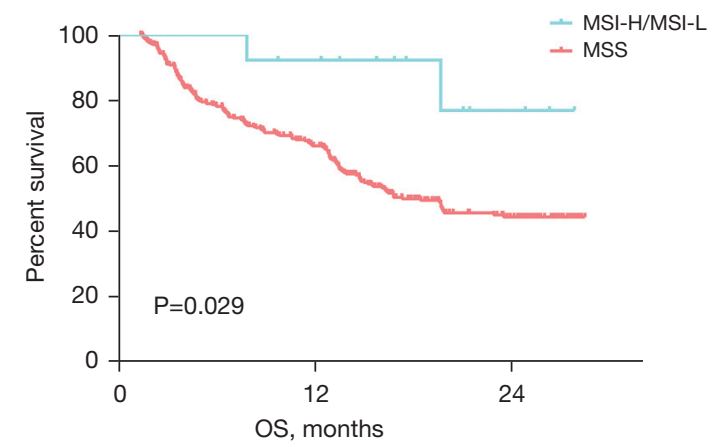

Figure 2 The Kaplan-Meier estimate of OS in patients with MSI or MSS gastric cancer. MSI, microsatellite instability; MSS, microsatellite stabilization; OS, overall survival.
Thompson et al. reported that GCs with higher $\mathrm{CD} 8^{+} \mathrm{T}$ cell densities also had higher PD-L1 expressions (26). Clear cell ovarian cancer (CCOC) patients with MSI showed higher $\mathrm{CD}^{+}$TIL and PD-1 ${ }^{+}$TIL numbers as well as an elevated $\mathrm{CD}^{+} / \mathrm{CD}^{+}$ratio. It is worth noting that $\mathrm{PD}$ L1 expression in tumor cells or immune cells occurred in all CCOC patients with MSI (40). However, in our study, patients with CD8 expression accounted for $95.2 \%$, and those with PD-L1 expression accounted for $2.5 \%$ of the total sample. The relationship between PD-L1 and CD8 expression indicated that GC patients with PD-L1 expression may be a subtype of patients with $\mathrm{CD} 8^{+}$rather than CD8-. This may be because PD-L1 is only highly expressed in some patients with cancer. Except for PD-L1, other immune regulators also exert biological functions in the tumor microenvironment. For example, recent studies have shown that CD47 (41), FGL1 (42), and Siglec-15 (43), which belong to immunoregulatory factors in the microenvironment, are independent of the PD-1/PD-L1 pathway. Although our findings showed that there was no significant difference in the expression level of PD-L1 in Chinese GC patients with MSI and GC patients with MSS, the present results seemed to indicate that the expression of CD8 and PD-L1 were mutually exclusive in Chinese MSI GC patients, suggesting that the MSI status of GC patients in China may help differentiate the immunotypes of GC.

\section{Conclusions}

As the first study to investigate the association between MSI

Table 3 The results of PD-L1 and CD8 expression in gastric cancers with MSI/MSS

\begin{tabular}{|c|c|c|c|c|c|c|c|c|c|}
\hline Characteristic & Total $(n=377)$ & \multicolumn{4}{|c|}{ PD-L1 expression } & \multicolumn{4}{|c|}{ CD8 expression } \\
\hline MSI & 13 & 0 & 13 & 0.443 & 0.506 & 13 & 0 & 0.675 & 0.411 \\
\hline MSS & 364 & 12 & 352 & & & 346 & 18 & & \\
\hline
\end{tabular}

MSI, microsatellite instability; MSS, microsatellite stabilization.

Table 4 The relationship between PD-L1 and CD8 expression in gastric cancers

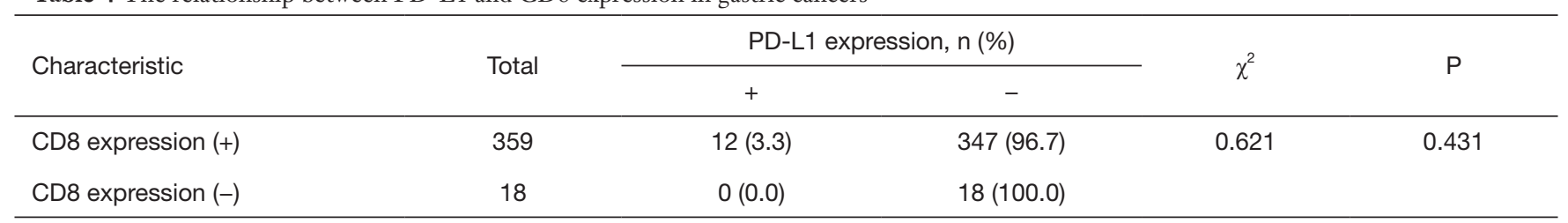

+ , positive; -, negative. 
and CD8 in GC, our results showed that the prevalence of MSI in GC differed significantly according to gender and age. Additionally, compared to patients with MSS, patients with MSI-H / MSI-L had a longer survival, and MSI expression was significantly correlated with CD8. However, the relatively small size of our MSI-H sample is not sufficient to draw firm conclusions, and further studies based on a larger cohort of GC samples are needed.

\section{Acknowledgments}

The authors thank Shanghai Tongshu Biotechnology Co. Ltd. for their technical support.

Funding: This work was supported by grant from National Natural Science Foundation of China (No. 82172941) and Henan Province Medical Science and Technology Research Project (No. 2018021018).

\section{Footnote}

Reporting Checklist: The authors have completed the REMARK reporting checklist. Available at https://dx.doi. org/10.21037/jgo-21-695

Data Sharing Statement: Available at https://dx.doi. org/10.21037/jgo-21-695

Conflicts of Interest: All authors have completed the ICMJE uniform disclosure form (available at https://dx.doi. org/10.21037/jgo-21-695). All authors acknowledge that this work received technical support from Shanghai Tongshu Biotechnology Co. Ltd. Chunxiao Wang is from Shanghai Tongshu Biotechnology Co. Ltd. The authors have no other conflicts of interest to declare.

Ethical Statement: The authors are accountable for all aspects of the work in ensuring that questions related to the accuracy or integrity of any part of the work are appropriately investigated and resolved. The study was conducted in accordance with the Declaration of Helsinki (as revised in 2013). This study was approved by the Ethics Committee of The First Hospital of Quanzhou (No. 2020126). All participants provided written informed consent.

Open Access Statement: This is an Open Access article distributed in accordance with the Creative Commons Attribution-NonCommercial-NoDerivs 4.0 International License (CC BY-NC-ND 4.0), which permits the non- commercial replication and distribution of the article with the strict proviso that no changes or edits are made and the original work is properly cited (including links to both the formal publication through the relevant DOI and the license). See: https://creativecommons.org/licenses/by-nc-nd/4.0/.

\section{References}

1. Bray F, Ferlay J, Soerjomataram I, et al. Global cancer statistics 2018: GLOBOCAN estimates of incidence and mortality worldwide for 36 cancers in 185 countries. CA Cancer J Clin 2018;68:394-424.

2. Bang YJ, Kim YW, Yang HK, et al. Adjuvant capecitabine and oxaliplatin for gastric cancer after D2 gastrectomy (CLASSIC): a phase 3 open-label, randomised controlled trial. Lancet 2012;379:315-21.

3. Cunningham D, Allum WH, Stenning SP, et al. Perioperative chemotherapy versus surgery alone for resectable gastroesophageal cancer. N Engl J Med 2006;355:11-20.

4. Macdonald JS, Smalley SR, Benedetti J, et al. Chemoradiotherapy after surgery compared with surgery alone for adenocarcinoma of the stomach or gastroesophageal junction. N Engl J Med 2001;345:725-30.

5. Sasako M, Sakuramoto S, Katai H, et al. Five-year outcomes of a randomized phase III trial comparing adjuvant chemotherapy with $\mathrm{S}-1$ versus surgery alone in stage II or III gastric cancer. J Clin Oncol 2011;29:4387-93.

6. Aoyama T, Yoshikawa T, Watanabe T, et al. Survival and prognosticators of gastric cancer that recurs after adjuvant chemotherapy with S-1. Gastric Cancer 2011;14:150-4.

7. Lee J, Lim DH, Kim S, et al. Phase III trial comparing capecitabine plus cisplatin versus capecitabine plus cisplatin with concurrent capecitabine radiotherapy in completely resected gastric cancer with D2 lymph node dissection: the ARTIST trial. J Clin Oncol 2012;30:268-73.

8. Sakuramoto S, Sasako M, Yamaguchi T, et al. Adjuvant chemotherapy for gastric cancer with S-1, an oral fluoropyrimidine. N Engl J Med 2007;357:1810-20.

9. Lindblad A, Kaucher S, Jaehn P, et al. The Incidence of Intestinal Gastric Cancer among Resettlers in GermanyDo Resettlers Remain at an Elevated Risk in Comparison to the General Population? Int J Environ Res Public Health 2020;17:9215.

10. Wang L, Li C, Tian J, et al. Genome-wide transcriptional analysis of Aristolochia manshuriensis induced gastric carcinoma. Pharm Biol 2020;58:98-106.

11. Cancer Genome Atlas Research Network. Comprehensive 
molecular characterization of gastric adenocarcinoma. Nature 2014;513:202-9.

12. Sohn BH, Hwang JE, Jang HJ, et al. Clinical Significance of Four Molecular Subtypes of Gastric Cancer Identified by The Cancer Genome Atlas Project. Clin Cancer Res 2017;23:4441-9.

13. Carethers JM. High predictability for identifying Lynch syndrome via microsatellite instability testing or immunohistochemistry in all Lynch-associated tumor types. Transl Cancer Res 2019;8:S559-63.

14. Han X, Zhang S, Zhou DC, et al. MSIsensor-ct: microsatellite instability detection using cfDNA sequencing data. Brief Bioinform 2021;22:bbaa402.

15. Choi YY, Kim H, Yang HK, et al. Clinical impact of microsatellite instability in patients with stage II and III gastric cancer: Results from the CLASSIC trial. J Clin Oncol 2017;35:4022.

16. Smyth EC, Wotherspoon A, Peckitt C, et al. Mismatch Repair Deficiency, Microsatellite Instability, and Survival: An Exploratory Analysis of the Medical Research Council Adjuvant Gastric Infusional Chemotherapy (MAGIC) Trial. JAMA Oncol 2017;3:1197-203.

17. Miceli R, An J, Di Bartolomeo M, et al. Prognostic Impact of Microsatellite Instability in Asian Gastric Cancer Patients Enrolled in the ARTIST Trial. Oncology 2019;97:38-43.

18. Pietrantonio F, Miceli R, Raimondi A, et al. Individual Patient Data Meta-Analysis of the Value of Microsatellite Instability As a Biomarker in Gastric Cancer. J Clin Oncol 2019;37:3392-400.

19. Chang L, Chang M, Chang HM, et al. Microsatellite Instability: A Predictive Biomarker for Cancer Immunotherapy. Appl Immunohistochem Mol Morphol 2018;26:e15-21.

20. Nassar AH, Mouw KW, Jegede O, et al. A model combining clinical and genomic factors to predict response to $\mathrm{PD}-1 / \mathrm{PD}-\mathrm{L} 1$ blockade in advanced urothelial carcinoma. Br J Cancer 2020;122:555-63.

21. Bang YJ, Kang YK, Catenacci DV, et al. Pembrolizumab alone or in combination with chemotherapy as firstline therapy for patients with advanced gastric or gastroesophageal junction adenocarcinoma: results from the phase II nonrandomized KEYNOTE-059 study. Gastric Cancer 2019;22:828-37.

22. Da Niel VC, Wainberg Z, Charles SF, et al. LBA009KEYNOTE-059 cohort 3: safety and efficacy of pembrolizumab monotherapy for first-line treatment of patients (pts) with PD-L1-positive advanced gastric/ gastroesophageal (G/GEJ) cancer. Ann Oncol 2017;28. doi: 10.1093/annonc/mdx302.008.

23. Bang YJ, Muro K, Fuchs CS, et al. KEYNOTE-059 cohort 2: Safety and efficacy of pembrolizumab (pembro) plus 5-fluorouracil (5-FU) and cisplatin for first-line (1L) treatment of advanced gastric cancer. J Clin Oncol 2017;35:4012.

24. Song Y, Gu Y, Hu X, et al. Endometrial Tumors with MSI-H and dMMR Share a Similar Tumor Immune Microenvironment. Onco Targets Ther 2021;14:4485-97.

25. Tokunaga R, Xiu J, Johnston C, et al. Molecular Profiling of Appendiceal Adenocarcinoma and Comparison with Right-sided and Left-sided Colorectal Cancer. Clin Cancer Res 2019;25:3096-103.

26. Thompson ED, Zahurak M, Murphy A, et al. Patterns of PD-L1 expression and CD8 $T$ cell infiltration in gastric adenocarcinomas and associated immune stroma. Gut 2017;66:794-801.

27. Derks S, de Klerk LK, Xu X, et al. Characterizing diversity in the tumor-immune microenvironment of distinct subclasses of gastroesophageal adenocarcinomas. Ann Oncol 2020;31:1011-20.

28. Fujiyoshi K, Yamamoto G, Takenoya T, et al. Metastatic Pattern of Stage IV Colorectal Cancer with HighFrequency Microsatellite Instability as a Prognostic Factor. Anticancer Res 2017;37:239-47.

29. Zhu H, Li X, Zhang X, et al. Polymorphisms in mismatch repair genes are associated with risk and microsatellite instability of gastric cancer, and interact with life exposures. Gene 2016;579:52-7.

30. Zhao P, Li L, Jiang X, et al. Mismatch repair deficiency/ microsatellite instability-high as a predictor for antiPD-1/PD-L1 immunotherapy efficacy. J Hematol Oncol 2019;12:54.

31. Zhu L, Li Z, Wang Y, et al. Microsatellite instability and survival in gastric cancer: A systematic review and metaanalysis. Mol Clin Oncol 2015;3:699-705.

32. Huang YQ, Yuan Y, Ge WT, et al. Comparative features of colorectal and gastric cancers with microsatellite instability in Chinese patients. J Zhejiang Univ Sci B 2010;11:647-53.

33. Cui M, Li P, Mao Y, et al. Implication of Microsatellite Instability in Chinese Cohort of Human Cancers. Cancer Manag Res 2020;12:10287-95.

34. Zang YS, Dai C, Xu X, et al. Comprehensive analysis of potential immunotherapy genomic biomarkers in 1000 Chinese patients with cancer. Cancer Med 2019;8:4699-708.

35. Beghelli S, de Manzoni G, Barbi S, et al. Microsatellite instability in gastric cancer is associated with better prognosis 
in only stage II cancers. Surgery 2006;139:347-56.

36. Arai T, Matsuda Y, Aida J, et al. Solid-type poorly differentiated adenocarcinoma of the stomach: clinicopathological and molecular characteristics and histogenesis. Gastric Cancer 2019;22:314-22.

37. Díaz Del Arco C, Estrada Muñoz L, Molina Roldán E, et al. Immunohistochemical classification of gastric cancer based on new molecular biomarkers: a potential predictor of survival. Virchows Arch 2018;473:687-95.

38. Ratti M, Lampis A, Hahne JC, et al. Microsatellite instability in gastric cancer: molecular bases, clinical perspectives, and new treatment approaches. Cell Mol Life Sci 2018;75:4151-62.

39. Kim ST, Cristescu R, Bass AJ, et al. Comprehensive molecular characterization of clinical responses to PD-1 inhibition in metastatic gastric cancer. Nat Med 2018;24:1449-58.

Cite this article as: Li X, Zhang L, Wang C, Huang J, Zhu J, Huang Y, Chen Y, Jia J, Jiang G. Microsatellite instability in Chinese gastric cancer and its correlation with clinical characteristics. J Gastrointest Oncol 2021;12(6):2719-2727. doi: 10.21037/jgo-21-695
40. Howitt BE, Strickland KC, Sholl LM, et al. Clear cell ovarian cancers with microsatellite instability: A unique subset of ovarian cancers with increased tumorinfiltrating lymphocytes and PD-1/PD-L1 expression. Oncoimmunology 2017;6:e1277308.

41. Matlung HL, Szilagyi K, Barclay NA, et al. The CD47SIRP $\alpha$ signaling axis as an innate immune checkpoint in cancer. Immunol Rev 2017;276:145-64.

42. Wang J, Sanmamed MF, Datar I, et al. Fibrinogen-like Protein 1 Is a Major Immune Inhibitory Ligand of LAG3. Cell 2019;176:334-347.e12.

43. Wang J, Sun J, Liu LN, et al. Siglec-15 as an immune suppressor and potential target for normalization cancer immunotherapy. Nat Med 2019;25:656-66.

(English Language Editor: D. Fitzgerald) 


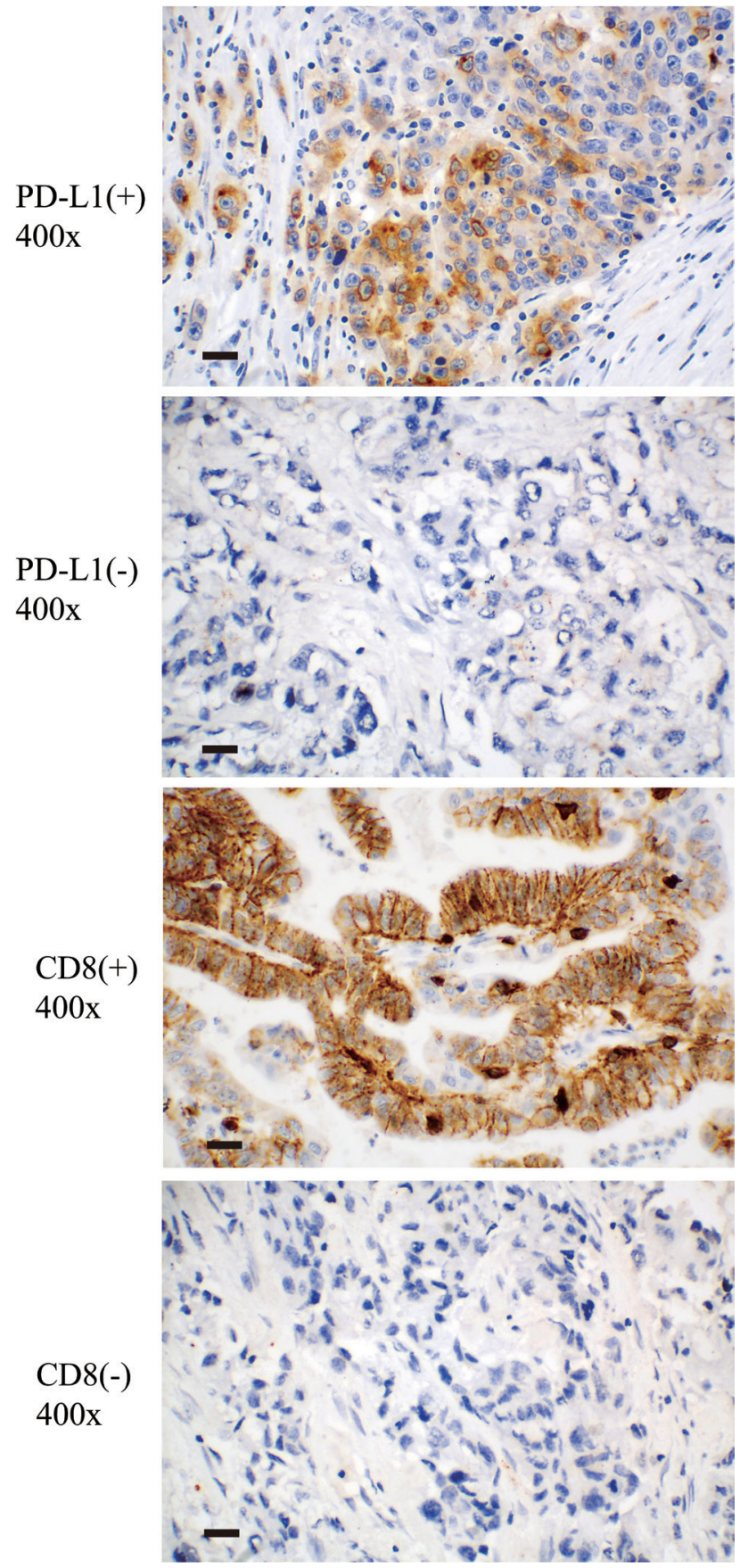

Figure S1 Representative images of PD-L1 and CD8 detection of gastric cancer tumor specimens (400x). CD8 and PD-L1 (SP142) antibodies were diluted at a ratio of 1:50 and incubated overnight at $4{ }^{\circ} \mathrm{C}$. After PBS cleaning, biotin-labeled secondary antibody (1:100) was dropped and incubated at $37^{\circ} \mathrm{C}$ for 30 minutes. $\mathrm{DAB}$ was used to develop the color for $15 \mathrm{~min}$ and rinse with tap water, then hematoxylin was redyed, and the tablets were sealed with xylene transparent resin, and observed and photographed under light microscope. 